\title{
The importance of play behavior for dog puppies
}

\author{
Olga Yudina $^{1}$, Anna Schavel $^{2}$, Anna Sipacheva ${ }^{1}$, and Irina Kalinina ${ }^{1}$ \\ ${ }^{1}$ Russian State Agrarian Correspondence University, 50, street highway Enthusiasts, Balashikha, 143900, Russia \\ ${ }^{2}$ Cynological Center, ownership 2/3, street highway Enthusiasts, Balashikha, 143915, Russia
}

\begin{abstract}
The study of the influence of game behavior on the mental state of service dogs' puppies of the German and Belgian (Malinois) shepherd breeds was conducted. The experimental and control groups included puppies of different ages - from 1-2 months to 3-6 months. The puppies of the experimental groups were trained with the use of educational games for puppies and young dogs, the puppies of the control groups did not have such training. As a result of a 6-month study, it was proved that the puppies of the younger experimental group showed the best results in 2 of the 4 tests. And the puppies of the older experimental group showed the best results in all 4 tests, in comparison with the results of the initial testing and the results of the control group.
\end{abstract}

\section{Introduction}

A dog is a highly organized animal with complex and diverse behavior. The concept of "behavior" is interpreted as the whole sum of the relationship of the organism with the environment. Moreover, the degree of complexity of behavior depends on the degree of complexity of the central nervous system, which has developed in the process of evolution [4,5]. In other words, the success of raising a puppy depends on the conditions in which its growth and development takes place. If these conditions are favorable, it will successfully lend itself to education and initial training. During the childhood, the dog must establish complex social connections with its own species, no less complex connections with another species, and become familiar with the main features of the environment, i.e., socialize $[1,2]$. Game behavior provides training for other forms of behavior; it is typical for the development of the body. In the game you can identify common signs of hunting, social behavior, and sexual activity. The game is a training for the development of the muscular, musculoskeletal, respiratory, cardiovascular and nervous systems. In addition, the game is necessary for the development of not only the body, but also the brain structures of the body [2, 3]. It should be noted that the relevance of studying the forms of game behavior helps to optimize the ways of influencing the dog, correctly interprets its behavior, which greatly facilitates the relationship between the dog and the person.

\section{Materials and methods}

The object of the study for 6 months was puppies and young dogs up to 12 months of age of service breeds (German $(\mathrm{n}=10)$ and Belgian (Malinois) $(\mathrm{n}=10)$ sheepdogs of the ODON Cynological Center in Balashikha. Each group had 5 males and 5 females. The age of the dogs involved in the study ranged from 1 to 12 months. The population of dogs was divided into the following age groups: puppies of the younger group (from 1 to 2 months) and puppies of the older group (3 to 6 months).

By randomization, the population of dogs was divided into experimental and control groups. The experimental group consisted of 10 heads of puppies of different ages: the Belgian shepherd - 3 heads (3-4 months of age) and 2 heads (at the age of 1 - 2 months), the German Shepherd-3 heads (1-2 months) and 2 heads (4-5 months). The control group also consisted of 10 puppies of different ages: the Belgian Shepherd - 3 heads (3 - 4 months) and 2 (1 - 2 months), the German Shepherd -3 individuals $(1-2$ months) and 2 (4 - 5 months).

With the puppies of the experimental groups, the games had beer held twice a week for six months. At the age of 1-2 months, it was a "Grab Toy" - rubber balls, hoses, a canvas "sleeve", etc., the acquaintance with the properties of household items, while the owner shows and names the object, explaining what they have to do with the dog and what you can do with them. The following games were played with the puppies of the experimental group at the age of 3-6 months: "Permission to bite", "Playful struggle", and the game with animals between the individuals of the experimental group of these breeds was observed. In this case, the intervention occurred only in a dangerous situation: "Animated" toys, "Chase" for a toy", "Search games (search of the room)", "Ladushki", "Hide and Seek", "Interception", "Search games (search of the area)", "Apportirovka". These games continued throughout the experiment, in order to strengthen contact with the puppies. When the puppies of the experimental group reached the age of six months, the games also began to be put into practice in various disciplinary exercises, it was used "switching" from excitement to braking and back; exercises on various shells and simulators, such games as "show what you want". The studies were carried out with usage of the developed and approved tests of Krivolapchuk Yu.V. [3]. All tests were carried

\footnotetext{
* Corresponding author: Udinich1977@yandex.ru
} 
out with the help of assistants who are not the owners of these dogs.

\section{Results and Discussion}

The initial testing of puppies at the age of 1-2 months included 2 tests: "Keys" and "Bait". During the "Keys" test, each puppy was brought to a separate area, without strong stimuli that interfere with the experiment. The puppy was placed next to the experimenter and a bunch of keys was thrown in the direction of the puppy slightly to the side. Two reactions were evaluated: desirable (positive - cautious research reaction) and weakly expressed (negative - pronounced reaction of avoiding this subject). According to this test, the puppies of the control group showed an $80 \%$ positive reaction, the puppies of the experimental group did not have a positive reaction.

The second test "Call for bait" was supposed to demonstrate the interest of the puppy in the feed. The desired reaction was that, according to the conditional signal, the puppies approached both the experimenter and the" master", the undesirable - a weak reaction to the food and an approach only to the "master". The test results revealed a $100 \%$ positive response in the control group and in the experimental group-only in $20 \%$ of cases. For older puppies, in addition to the "Keys" test, there are tests for the attitude to the person "Person" (familiar and unfamiliar); for determining the development of social stereotypes of puppies by their behavior-the "Landing" test and the final test for determining the development of the puppy's psyche "Communication".

In the "Keys" test, three reactions were identified: desirable (positive), weakly expressed (negative), and the absence of any reaction. In the "Human" test, two reactions were revealed: desirable (positive - a clear difference between "their" and "others") and weakly expressed (negative - a weak understanding of the difference between "their" and "others"). The "Landing" test implies desirable behavior (positive-manifested in the puppy's attempt to imitate the cage on the owner's leg, which proves the normal development of social stereotypes), the opposite reaction was the absence of the puppeis attempt to fill up the croup on the owner's leg and a weak attempt (negative behavior). The test "Communication" was characterized by 2 forms of behaviors. Desirable (positive) one consisted in confident behavior in the absence of older dogs and showing respect up to fawning in their presence. It is also desirable if the puppy shows a connection with the owner, appeals to him with a request for protection. Undesirable (negative) one is when the puppy treats the adult individual with complete indifference and tries to dominate it.

In the control group, the animals showed $100 \%$ positive behavior in all tests. In the experimental test the test "Keys" - in $20 \%$ of cases was negative, and in $80 \%$ of cases there was no reaction. The test "Person" was $100 \%$ negative reaction; the test "Landing" - 20\% positive reaction, $40 \%$ - negative and no reaction; the test "Communication" - $60 \%$ positive and $40 \%$ negative reaction.

After 6 months, the final testing of the puppies of the experimental groups at the age of 6-7 months was carried out (Fig. 1). However, the content and interpretation of the tests had changed. In the first test, "Keys", we identified only undesirable behavior of animals; it was expressed in the fact that the puppies of both groups $(100 \%)$ did not pay any attention to the experimenter and showed unreasonable curiosity about the subject.

The second test was to determine the relationship to the person (familiar and unfamiliar). The desired behavior is with acceptable caution in relation to the" stranger", as well as a good level of contact with the experimenter, up to the request for permission to approach a stranger. A positive reaction was observed in $100 \%$ of cases in the experimental group and only $20 \%$ in the control group. A variant of undesirable (negative) behavior of puppies manifested in complete indifference to a stranger that was also identified - the control group $(40 \%)$.

The desired (positive) behavior in the "Landing" test was manifested in the puppy's attempt to imitate the cage on the owner's leg, which proves the normal development of social stereotypes. This reaction was shown by 4 puppies of the experimental group. The negative reaction in the control group was $60 \%$, and in the experimental group was $20 \%$. The puppies needed more time to land, and they also rested on the body of the body, without piling the croup on the owner's leg.

The opposite reaction was the absence of an attempt -4 puppies in each group (40\%) filled up the croup on the owner's leg, landing at a distance from the experimenter. Thus, the author of the test N. D. Krivolapchuk said about the lag in the development of the psyche of puppies. The final test for determining the development of the psyche of puppies of 6-7 months was the test of intraspecific communication. Recorded one form of manifestation is a variant of the desired reaction (positive). All the puppies demonstrated confident behavior until the older individual dominated them. Then the puppies made an appeal to the owner with a request for protection.

The final testing of puppies aged 9-12 months (Fig. 2) showed in the test "Keys" the desired reaction - the dog carefully observes the experimenter's hand. Then they switches all attention to the abandoned bunch of keys and after the examining object. Then the attention to the experimenter himself manifested in $80 \%$ of individuals in the experimental and only $20 \%$ - in the control groups. In the "Person" test, the desired reaction (a clear distinction between "your" and "others'") was found in $100 \%$ of the experimental and $60 \%$ - of the control group.

At this age, in the "Landing" test, the desire to make a cage on the owner's leg must be absent. A positive reaction was shown by $60 \%$ of the puppies of the experimental and $40 \%$ of the control groups. There was a variant of undesirable behavior (negative), it was shown by $40 \%$ of the control and $20 \%$ of the experimental groups. In the "Communication" test, only one form of 
reaction was identified - desirable (positive). It was shown by all $100 \%$ of individuals, consisting in confident behavior, as well as permissible attempts to dominate an adult dog and terminated in the event of a protest by the trainer (owner).

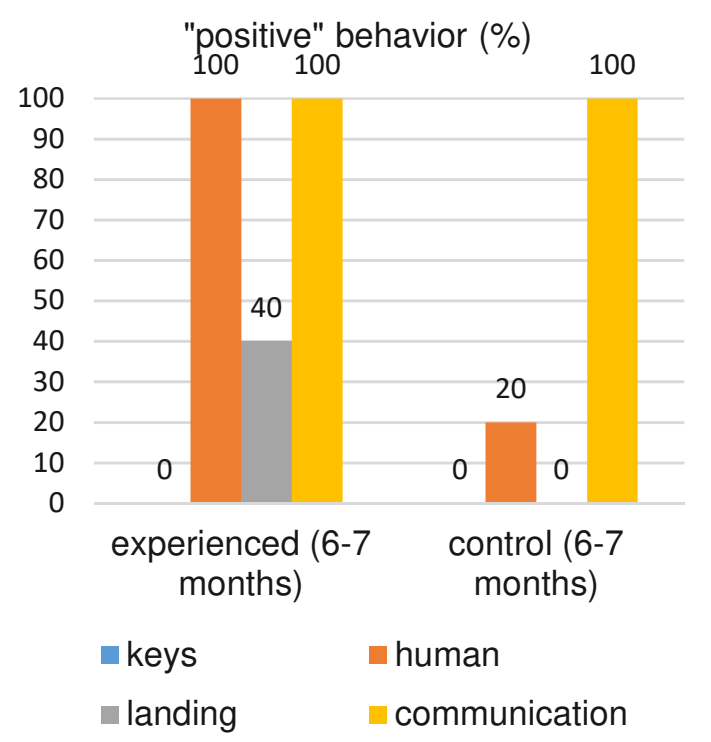

Fig. 1 Final testing of puppies of experimental groups at the age of 6-7 months

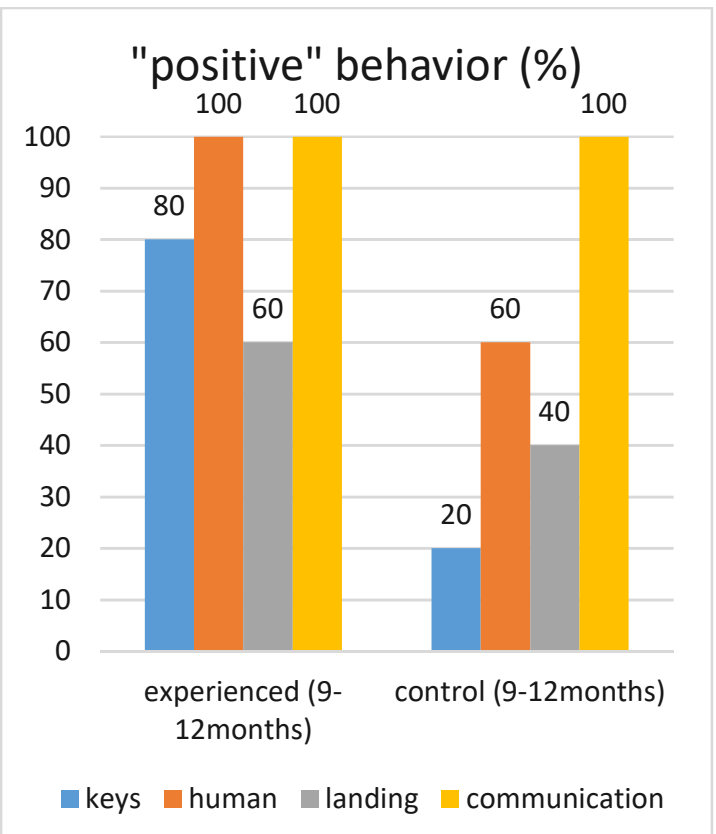

Fig. 2. Final testing of puppies of experimental groups aged 9-12 months

The analysis of changes in the psychological development of puppies from one month to seven months of the experimental and control groups, as well as from 3 to 12 months (Fig. 3) showed that the puppies of the experimental group, with whom they were purposefully and systematically engaged, ultimately showed results higher by 5 points compared to the results of the initial testing. And the control group, on the contrary, lowered their results by 6 points.

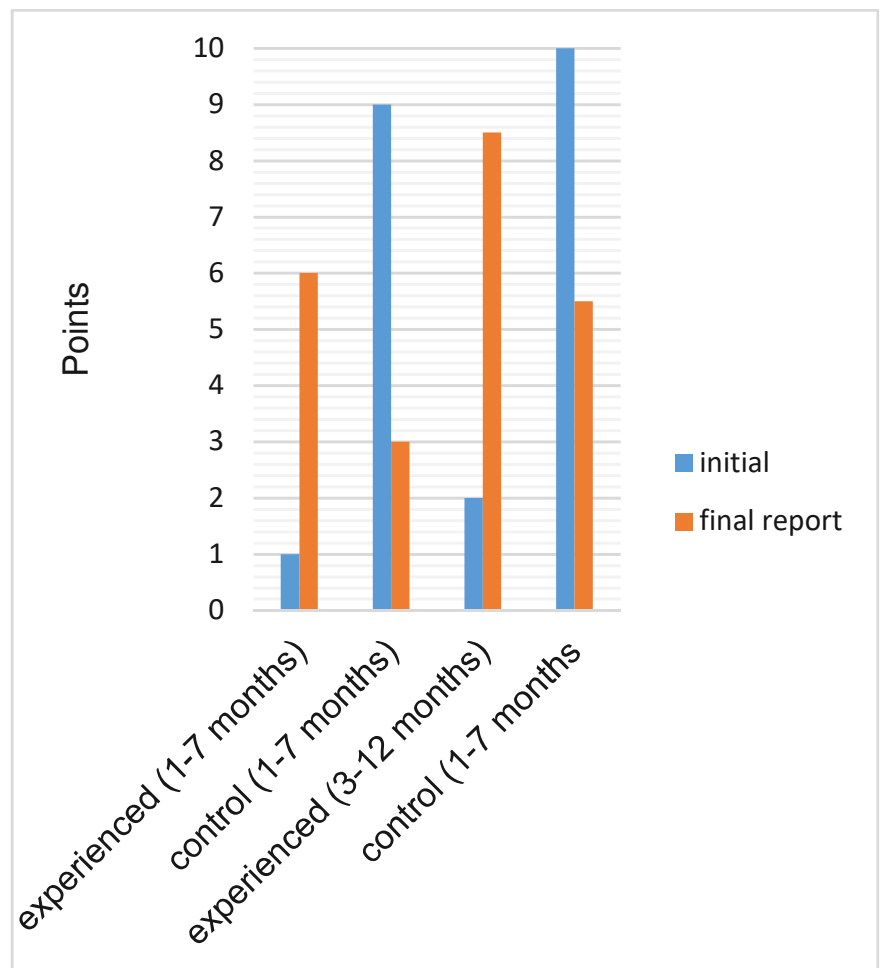

Fig. 3. Analysis of changes in the psychological development of experimental groups

In the final test (Fig. 3), the individuals of the older experimental group from 3 to 12 months showed results of 6.5 points $(65 \%)$ higher than in the initial test. And the puppies of the control group, at the final testing, showed results 4.5 points ( $45 \%$ ) lower than the results of the initial testing.

Analysis of changes in the psychological development of German and Belgian Shepherd puppies from three to six months to nine to twelve months of the experimental group (Fig. 4) revealed that the relative result of the Belgian Shepherd improved by $12.5 \%$, in German shepherds by $31.25 \%$. The results of the final testing among different breeds were almost unchanged ( $0 \%$ and $3.72 \%$, respectively).

As part of the study, we conducted a survey of dog owners, divided into two groups: the first "Dog handlers" - those who receive or have professional training, and the second-owners of pets. The survey was aimed at determining the level of human-dog contact and determining the value of games in raising a puppy. The group of dog handlers pays more attention to games with dogs. Both with puppies and with adult dogs (36.14 \pm 0.42$)$, pet owners do not attach so much importance to games and do not devote so much time to the game (31.67 \pm 0.66$)$ (Fig. 5). 


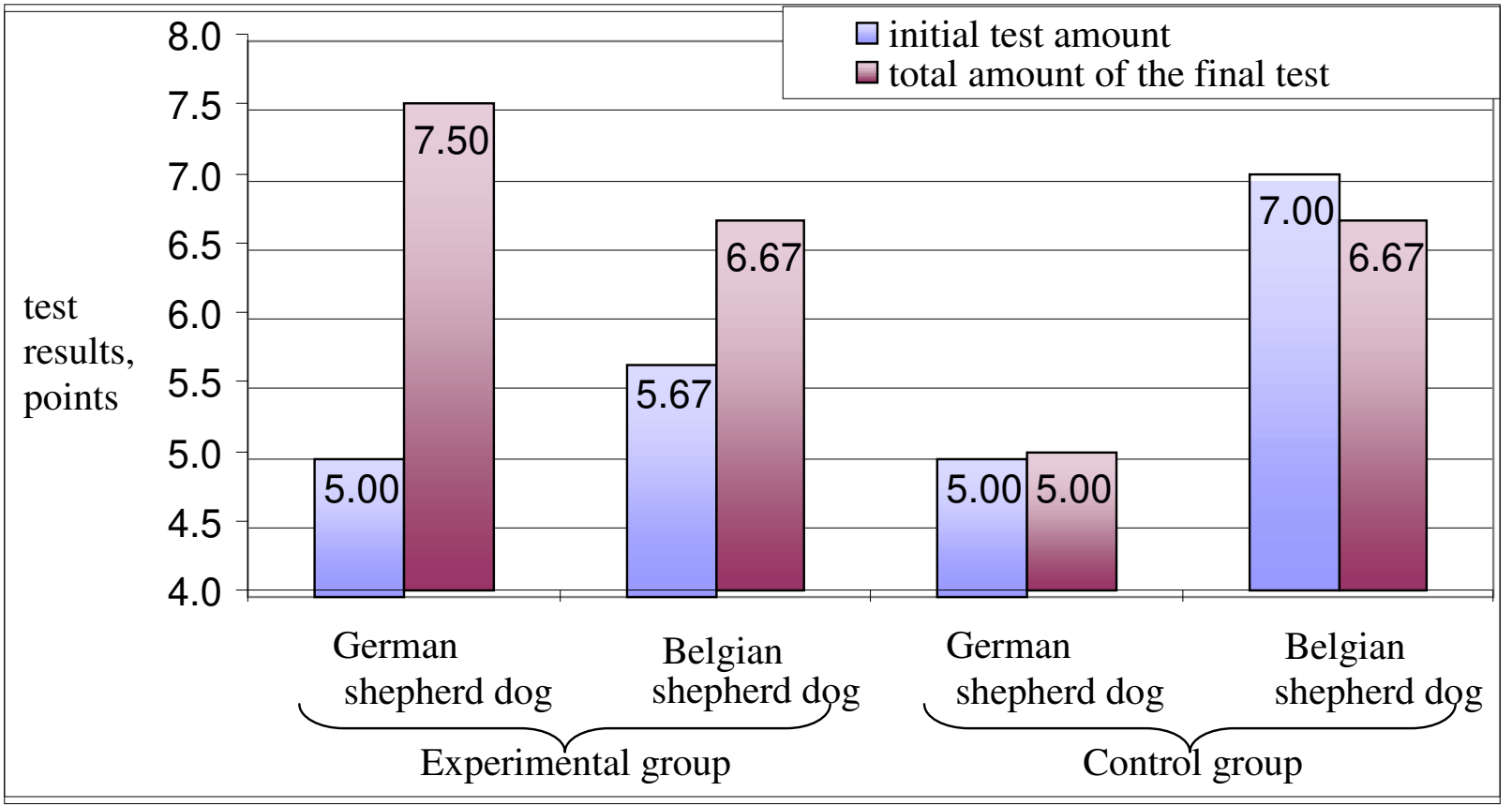

Fig. 4. Analysis of changes in the psychological development of German and Belgian Shepherd puppies starting from three to six months ending with nine to twelve months of the experimental and control groups.

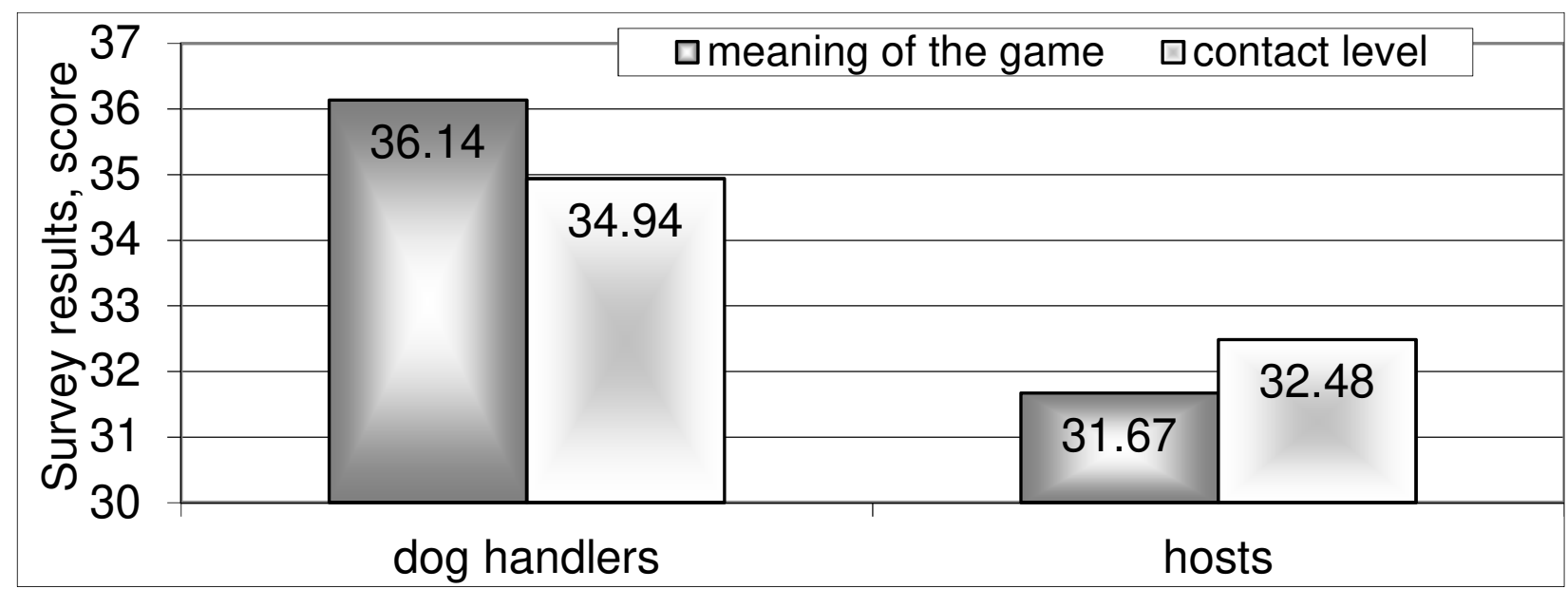

Fig. 5. The ratio of the value of the game for dog owners with the level of his contact with the dog.

Thus, education with the help of educational games during the experiment brought the puppies of the experimental group to better results than the puppies of the control group, which at the beginning of the experiment were leading in their psychological qualities. We must not forget that all the puppies during the experiment had communication with the trainer and the puppies of the control group were also under the influence of a person.

\section{Conclusions}

As a result of the done work, the following conclusions were made:

1) The puppies of the younger experimental group from 1 to 2 months at the initial testing showed weaker results ("Keys" - 0\% and "Bait» - 20\%, compared to the control group of $80 \%$ and $100 \%$, respectively). At the initial testing, the puppies of the experimental group at the age of 3-6 months showed positive results in two tests, but lower than in the control group (in the "Landing" test-20\% and 100\%, respectively; in the "Communication" test-60\% and $100 \%$, respectively).

2) After 6 months of the experiment, the puppies of the younger experimental group from 6 to 7 months showed higher results in two of the four tests in comparison with the results of the initial test and compared with the control group ("Man" - 100\%, 20\%, respectively; "Landing" - 40\%, 0\%, respectively). Puppies of the older experimental group from 9 to 12 months showed higher results in all four tests compared with the results of the initial testing, and compared with the control group ("Keys" - 80\%, 20\%; "Man" - 100\%, 60\%; "Landing" - 60\%, 40\%; "Communication" - 100\%, 100\%, respectively).

3) The analysis of the attitude of dog owners to developing games by the questionnaire method revealed the following results. Among dog handlers, $60.2 \%$ believe that the game is important; they spend 
much more time playing with the dog. Among the owners, only $39.5 \%$ pay enough attention to playing with the dog. Determination of the level of contact by the questionnaire method revealed that dog handlers $(58.2 \%)$ have a higher level of contact with the dog than owners do with their own animals (54.0\%).

\section{References}

1. V. N. Zubko Education of the puppy (1996)

2. E. N. Mychko, M. N. Sotskaya, V. A. Belenky Dog behavior: a manual for dog breeders, (2004)

3. Krivolapchuk, N. D. Applied psychology of domestic dogs: a textbook, (2008)

4. L. Chebykina, Understand a friend. Handbook of dog behavior, (2005)

5. N. B. Nikulina, Behavioral problems of dogs: an educational and methodological guide krost, (2016) 